\title{
Multimodal Face and Iris Recognition with Adaptive Score Normalization using Several Comparative Methods
}

\author{
Lemmouchi Mansoura, Athamena Noureddine, Ouarda Assas and Abdessemed Yassine
}

Department of Electronics, Faculty of Engineering, University of Batna 2, Rue Chahid Mohamed El Hadi Boukhlouf, Batna 05000,Algeria; lemmouchi2mansoura@gmail.com, atha_nour@yahoo.fr, assaa_warda@yahoo.fr, yabdes@yahoo.fr

\begin{abstract}
Objectives: To combine several biometric methods used for face and iris simultaneous recognition of an individual in order to enhance the performance. Methods/Analysis: For every methodology, four approaches are used for features extraction: discrete wavelet transform, singular value decomposition, discrete cosine transform and principal component analysis. Then, matching is employed by different distance measurements: City block, Euclidean, Seuclidien, Cosine, Chebychevand Correlation. Findings: The most widely used normalization method such as min-max and a new method using geometric mean is presented. The data fusion is performed at the score level with two methods: simple sum and weighted sum. The obtained comparison results show that PCA (face) and PCA (iris) fusion scenario associated with simple sum rule and the proposed new normalization method (geometric mean) have given the best recognition rate. Application/Improvements: This new normalization method helps considerably to enhance other methods in a multimodal biometric recognition system.
\end{abstract}

Keywords: Distances, Face, Fusion, Geometric Mean, Iris, Multimodal Biometrics, Recognition Rate

\section{Introduction}

Nowadays, with the new advances in digital technologies, security and access control are necessary demands. The security domain uses various authentication methods to keep information protected: the oldest and simplest form is based on password or name, followed by the smart card, and the latest method is biometrics which measures the physical or behavioral characteristics of people. It includes iris, face, hand engineering, fingerprints, palm pattern, blood vessel pattern, and personal behaviors such as signature, voice, and walking modes. Each of these attributes has its own characteristics that are consistent with the requirements of different security systems. Biometric systems can be divided into mono- and multimodal systems. A uni-modal biometric system is with one type of component based on a solitary methodology such as fingerprints, iris, face... while a multimodal system has higher than one type of component and provides high accuracy recognition.

With the limited accuracy in uni-modal biometric systems, many problems occur and among them:

- In facial recognition, illumination can affect the image quality, conditions and facial expressions.

- In fingerprint recognition, a scanner isn't able to read clearly either dirty fingerprints or the elderly faded fingerprints and young children underdeveloped fingerprints ridges and these lead to false database matches

- Difficulty to acquire iris images if the subject has a pathological eye condition.

Biometric systems' idea is to choose a reasonable biological trait for identification. Many researchers have opted for mono-modal biometric framework. $\mathrm{In}^{1}$ proposed a method of personal identification by analyzing highly 
stable and distinctive iris patterns. The researchers did use the wavelet transform to enhance the efficiency and accuracy of the proposed system, in order to make the feature vector compact and efficient, they had to initialize the weight vector and the winner selection. $\operatorname{In}^{2}$ used a bank of Gabor filters and introduced an iris recognition system who formed a fixed length feature by capturing both local and global iris characteristics, so the recognition speed is increased based on the metric Euclidean distance between the two corresponding iris vectors of the iris matching process. $\mathrm{In}^{3}$ proposed a system which utilizes the Active Appearance Model (AAM) called face recognition framework, in order to locate and segment primary facial features. To enhance the face recognition and retrieval performance, they utilized the detection of the facial marks is by the Laplacian-of-Gaussian and morphological operators. Using FERET and Mugshot the proposed system was tested. $\mathrm{In}^{4}$ focused on investigating automated face recognition framework utilizing the eigenfaces technique that is based on the artificial neural networks and the principle component analysis. Illumination, distance and subject's head orientation that influences the analyses on the face recognition system that was built for office door access control was included in this research. As in ${ }^{5}$, without compromising any security conflict in connection to the iris biometrics they endeavoured to address the ability to handle strong, accurate matching and unconstrained acquisition, and privacy upgrade. They had presented a unified iris system dependent on unsystematic projections and sparse representation in order to achieve that. Multimodal biometrics, which consists of combining several systems biometric, is more and more studied. Indeed, it makes it possible to reduce certain limitations of unimodal biometric systems, such as the impossibility of acquiring data from certain people or intentional fraud, while improving recognition performance. These benefits of multimodality to unimodal biometric systems are achieved by merging several biometric systems.

The advantages of using biometric multimodal are:

- The possibility of error tolerance because the system can work even if some biometric sources are unreliable

- Addresses the issue of non-universality faced by mono-biometric systems.

- Facilitate the filtering or indexing of vast-scale biometric databases.

- It is difficult to impersonate several biometric characteristics of a legally registered individual.
- Enables continuous monitoring or individual followup in situations where an attribute is insufficient.

- Effectively process noisy data.

Multimodal biometric systems are very difficult to usurp compared to unimodal systems. Even though a biometric modality may be usurped, the individual may still be authenticated using the other biometric identifier.

Through fusing two or three biometrics considering no rule for their selection, many researchers tried proposing multimodal biometric systems, so by fusing iris and face we can have a multimodal biometric framework. The selection of biometrics is through experiment and error because there are no guidelines for this, and that does not mean a misconception to all this research's and studies.

$\operatorname{In}^{6}$ developed a multimodal biometric approach by combining face and iris features. The proposed approach was validated and evaluates using an experimental multimodal biometric database; this database is a combination of an ORL face database and CASIA iris database contracted by the researchers. $\mathrm{In}^{7}$, based on a matching score level fusion and an improved speed-up version of the support vector machine. They presented a face-iris multimodal biometric framework. Utilizing a proposed feature selection method to choose the ideal subset of features the performances of face and iris recognition were enhanced. The introduced system did show very promising results after the experimental results are out. Also, in ${ }^{8}$, displayed a software-based fake detection method that can be utilized in multiple biometric frameworks to detect different types of fraudulent an access attempts for improving biometric recognition frameworks security through having aliveness assessment in a fast, user-friendly and nonintrusive. The proposed technique was tested and evaluated utilizing publicly available datasets of fingerprint, iris and face. Also, in ${ }^{9}$, the presented Multimodal Biometric frameworks: A Comparative Study exhibited and built up a near model dependent on PCA and Euclidian matcher utilizing MATLAB R2014b test system, SDUMLA-HMT and CASIA-FACEV5 databases to examine the execution of multimodal biometric systems utilizing both heterogeneous and homogeneous information combined by highlight level combination or score level combination. Also $^{10}$, introduced Prognostic assessment of multimodal biometric traits recognition dependent on human face, finger print and iris images using ensemble SVM classifier.

Biometric distinguishing proof systems that utilization or can utilize a blend of at least two biometric 
modalities to recognize an individual are called multimodal biometric frameworks. The most imperative explanation for the utilization of multimodal biometric frameworks is the change of the acknowledgement rate. In this study, we study the performance of several methods inclusive the feature extraction module utilizing principal component analysis (PCA), discrete wavelet transforms (DWT), singular value decomposition (SVD) and discrete cosine transform (DCT). Other methods in the classification unit (City block, Euclidean, Seuclidean, Cosine, Chebychev and Correlation), and two normalization techniques (Minmax and Geometric mean), data normalization is needed since the produced matching scores from the face and iris images are not homogenous. These scores are transformed into a common domain and range. The fusion of these two modalities is then performed using simple sum and weighted sum Rules. Experiments conducted on a database of 40 people show that the implementation of a multimodal framework relies upon on the choice of the normalization method and the fusion method. The proposed normalization method has given the best results. This study is organized as follows: section 2 presents a full description of proposed multimodal system for individual recognition, where each step of the system is developed in detail. Section 3 shows the obtained experimental results and discussions and section 4 concludes this study.

\section{Proposed Approach for Multimodal Recognition}

The main steps engaged in the proposed multimodal framework are; image acquisition, feature extraction, classification, normalization, fusion and decision.

\subsection{The Capture Module}

Is in charge of for acquiring the biometric data of an individual (this can be a camera, a fingerprint reader, a security camera, etc.). In this work, the pictures of face and iris are extricated from (Face Database ORL (Olivetti Research Laboratory). This database contains 40 people, where each person is represented by a set of 10 different images of size $48 \times 48$ ). And (CASIA IrisV1 database of Iris images includes 756 Iris photos of 108 people. For each person, 7 photos are captured by the camera developed by CASIA-IrisV1 which gets close to the Iris, where three samples (photos) are taken in the first session, and four in the second session. All photos are saved in BMP format with a resolution of " $320 \times 280$ ").

After this step, we move to the pre-processing step, we used only the size normalization to minimize the time.

\subsection{The Feature Extraction Module}

Takes as info the biometric data obtained by the capture module and extracts just the applicable information to form a new representation of the data. Ideally, this new representation is supposed to be unique for each person and relatively invariant to intra-class variations, the methods utilized for feature extraction are Discrete Cosine Transform ${ }^{11}$, Discrete Wavelet Transform ${ }^{12}$, Principal Component Analysis ${ }^{13}$, and Decomposition in singular values ${ }^{11}$.

\subsection{The Classification Module}

Compares the set of characteristics extracted with those of the model recorded in the framework database and decides the degree of similarity (or divergence) between the two. There are several methods for calculating the distance between multidimensional vectors. Here, six different distance measurements are used: City block, Euclidean, Seuclidean, Cosine, Chebychev and Correlation ${ }^{14}$.

\subsection{Fusion}

The combination of several biometric systems can be done at four different levels: at the data level, at the level of the characteristics extracted, at the level of the scores of the comparison module or at the level of decisions. The fusion at the level of the scores seems to be the best choice. The processing chain is presented in Figure 1 illustrates our choice of melting scores. Two rules of fusion are employed which are: the simple sum and the weighted sum, and defined as ${ }^{15}$ :

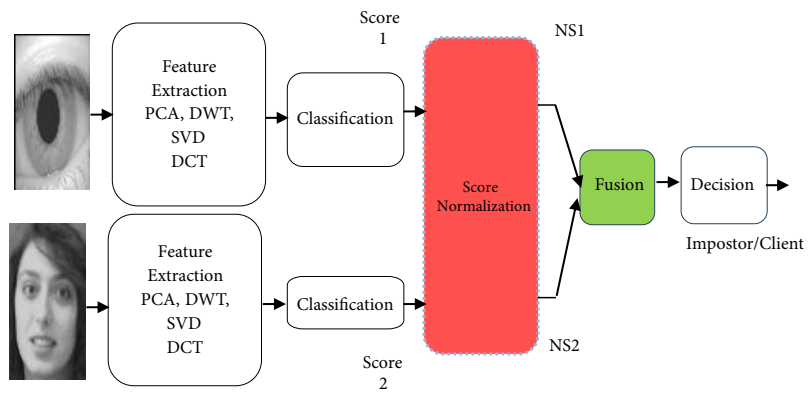

Figure 1. Multimodal Approach (NS: Normalized score). 


\subsection{The Decision Module}

Verifies the identity asserted by a user or decides the identity of an individual dependent on the degree of similarity between the characteristics extracted and the stored model(s).

\section{Discussion and Analysis of the Results}

The test and evaluation of implemented systems requires the creation of a multimodal corpus, this later consist of forty virtual individuals having two different modalities (face and iris) and extracted. from the face Database ORL (Olivetti Research Laboratory) and the iris database CASIA (China Institute of Automation). Three training images and three test images for each modality are taken for each person. The MATLAB software is used to implement this multimodal biometric framework.

The performance of the biometric framework is measured by rate recognition ( $R R)$ is determined by:

- Rate recognition RR is the measure of the likelihood that the biometric security

- Framework will incorrectly accept an access attempt by an approved user.

$$
\mathrm{RR}=\frac{\text { nombre of reconized face }}{\text { the size of database test }} \times 100 \%
$$

Three models of biometric systems have been implemented:

Model 1: the identification of the person is based on the modality face.

Model 2: the identification of the person is based on the modality iris.

Model 3: the identification of the person is based on the two modalities face and iris.

For each model, four methods PCA, DWT, SVD and DCT are used for extracting the features with six different classifiers based on different distances: (City block, Euclidean, Seuclidean, Cosine, Chebychev and Correlation).

The average was used to model the general behavior of the methods and distances implemented.

\subsection{Unimodal}

\section{- Model FACE}

Table 1 and Figure 2 demonstrate the comparison of face recognition between four methods PCA, DWT, SVD and
DCT using different distances (Euclidean, Seuclidean, Chebychev, City Blok, Cosine and Correlation). The PCA, DCT and SVD methods gave the best Data recognition rate $(91.67 \%)$ for the Euclidean distance. The SVD method gave the same result for the Seuclidean distances also.

Table 1. Rates of Face Recognition

\begin{tabular}{|l|c|c|c|c|}
\hline \multicolumn{5}{|c|}{ Rate recognition \% FACE } \\
\hline & PCA & DWT & SVD & DCT \\
\hline Euclidean & 91.67 & 86.67 & 91.67 & 91.67 \\
\hline Seuclidean & 75.83 & 80.00 & 91.67 & 77.50 \\
\hline Chebychev & 81.67 & 74.17 & 70.00 & 75.83 \\
\hline Cityblock & 90.00 & 88.33 & 90.83 & 89.17 \\
\hline Cosine & 86.67 & 80.83 & 86.67 & 86.67 \\
\hline Correlation & 85.83 & 80.83 & 86.67 & 86.67 \\
\hline Average & 85.28 & 81.80 & 86.25 & 84.59 \\
\hline
\end{tabular}

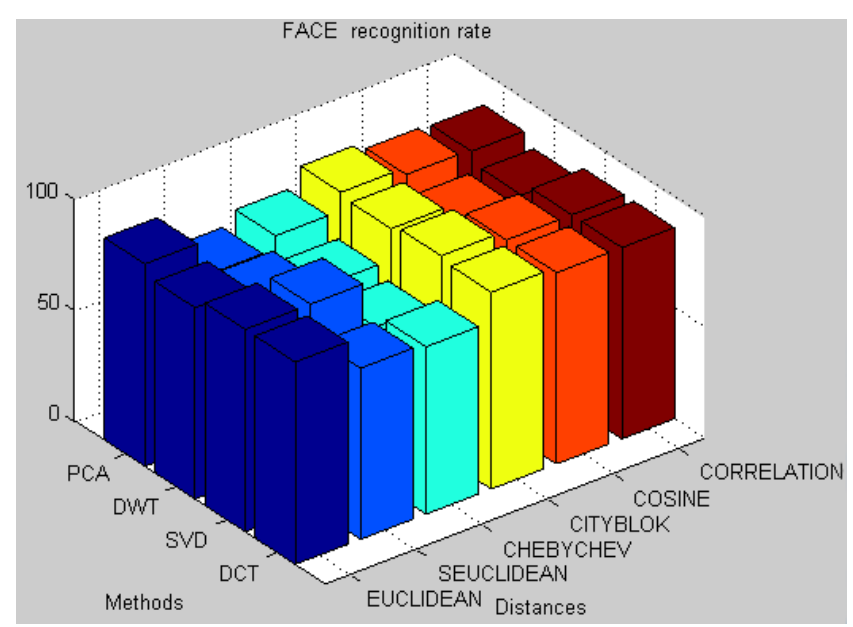

Figure 2. Histograms of obtained Results of Face recognition utilizing PCA, DWT, SVD and DCT utilizing different distances.

\section{- Model IRIS}

Table 2 and Figure 3 demonstrate the comparison of iris recognition between several methods. The PCA method gave. The best Data recognition rate $(81.67 \%)$ for the City blocks distance.

Table 2. Rates of good Iris Recognition

\begin{tabular}{|l|c|c|c|c|}
\hline \multicolumn{5}{|c|}{ Rate recognition \% IRIS } \\
\hline & PCA & DWT & SVD & DCT \\
\hline Euclidean & 77.50 & 76.67 & 40.00 & 77.50 \\
\hline Seuclidean & 75.00 & 80.83 & 44.17 & 57.50 \\
\hline Chebychev & 58.33 & 55.83 & 37.50 & 64.17 \\
\hline
\end{tabular}




\begin{tabular}{|l|l|l|l|l|}
\hline Cityblock & 81.67 & 80.83 & 45.83 & 70.00 \\
\hline Cosine & 72.50 & 72.50 & 45.83 & 72.50 \\
\hline Correlation & 75.00 & 69.17 & 43.33 & 72.50 \\
\hline Average & 73.33 & 72.63 & 42.78 & 69.29 \\
\hline
\end{tabular}

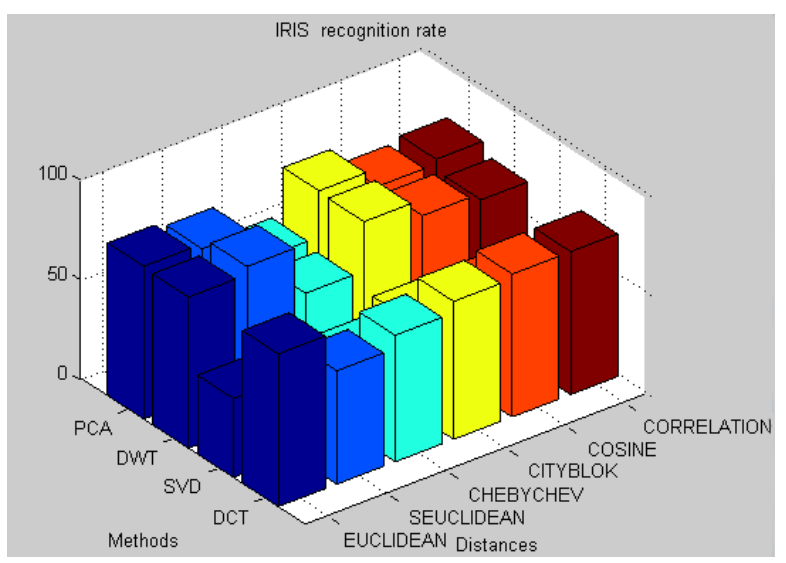

Figure 3. Histograms of obtained Results of Iris recognition utilizing PCA, DWT, SVD and DCT utilizing different distances.

\subsection{Multimodal}

In this section, we present the way to fusion the two modalities (face and iris) based on the appearance described previously. We give an outline of the proposed technique. The recognition is implemented by the following steps:

- Representation of the image of the iris and face of entry by the entities PCA (face) and PCA (iris), DWT (face) and DWT (iris), SVD (face) and SVD (iris), DCT (face) and DCT (iris)

- Vectors of measurement of distance and of the input image of all models of face and iris $\mathrm{N}$ stored in the database are calculated;

- The normalization of all distances for each class is required $\left(\min -\max ^{16}\right.$ and a new method Geometric mean ${ }^{17}$ )

The geometric mean of a sample $\mathrm{X}$ is

$$
m=\left[\prod_{i=1}^{n} x i\right]^{\frac{1}{n}}
$$

Where $n$ is the number of values in $\mathrm{X}$.

- Use multimodal fusion (simple sum, weighted sum).

Table 3 presents the assessment results of the multi-modal system using normalization (Min-Max) and using (simple sum) fusion rules. The PCA method gave the best Data rec- ognition rate (96.67\%) for the city block distance. Figure 4 shows the histograms obtained multimodal recognition rate (face + iris) using normalization (Min-Max) and using (simple sum) fusion rules. Table 4 presents the assessment results of the multi-modal system using normalization (Min-Max) and using (Weighted Sum) fusion rules. The PCA method gave the best Data recognition rate $(95.83 \%)$ for the city block distance. Figure 5 shows the histograms obtained multimodal recognition rate (face and iris) using normalization (Min-Max) and using (Weighted Sum) fusion rules. Table 5 presents the assessment results of the multi-modal system using normalization (Geometric mean) and using (simple sum) fusion rules. The PCA method gave the best Data recognition rate $(98.50 \%)$ for the city block distance. Figure 6 shows the histograms obtained multimodal recognition rate (face + iris) using normalization (Geometric mean) and using (simple sum) fusion rules.

Table 3. Obtained recognition rate of multimodal recognition (face + iris) utilizing normalization (MinMax) and utilizing fusion rules (simple sum)

\begin{tabular}{|l|c|c|c|c|}
\hline \multirow{2}{*}{\multicolumn{1}{c|}{ Rule }} & \multicolumn{4}{c|}{ Normalization (Min-Max) } \\
\cline { 2 - 5 } & \multicolumn{4}{c|}{ Simple Sum } \\
\cline { 2 - 5 } & PCA & DCT & DWT & SVD \\
\hline Euclidean & 94.17 & 94.17 & 92.50 & 91.67 \\
\hline Seuclidean & 89.17 & 79.17 & 93.33 & 89.17 \\
\hline Chebychev & 80.83 & 75.00 & 77.50 & 82.50 \\
\hline Cityblok & 96.67 & 93.33 & 94.17 & 94.17 \\
\hline Cosine & 91.67 & 89.17 & 84.17 & 89.17 \\
\hline Correlation & 92.50 & 91.67 & 89.17 & 88.33 \\
\hline Average & 90.83 & 87.09 & 88.48 & 89.17 \\
\hline
\end{tabular}

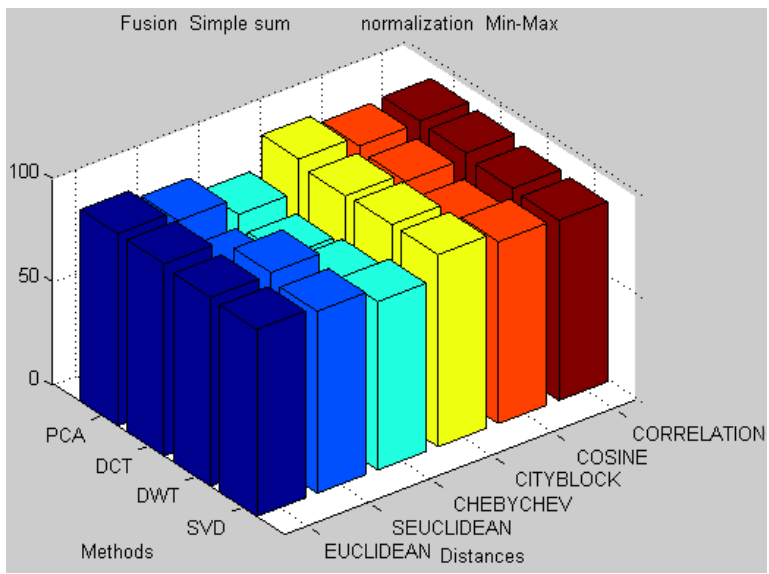

Figure 4. Histograms obtained multimodal recognition rate (face + iris) utilizing normalization (Min-Max) and utilizing (simple sum) fusion rules. 
Table 4. Obtained recognition rate of multimodal recognition (face + iris) utilizing normalization (MinMax) and utilizing fusion rules (Weighted sum)

\begin{tabular}{|l|c|c|c|c|}
\hline \multirow{2}{*}{ Rule } & \multicolumn{4}{|c|}{ Normalization (Min-Max) } \\
\cline { 2 - 5 } & \multicolumn{4}{|c|}{ Weighted Sum } \\
\cline { 2 - 5 } & PCA & DCT & DWT & SVD \\
\hline Euclidean & 95.00 & 95.83 & 93.33 & 92.50 \\
\hline Seuclidean & 91.67 & 87.50 & 91.67 & 91.67 \\
\hline Chebychev & 84.17 & 81.67 & 83.33 & 82.50 \\
\hline Cityblok & 95.83 & 95.00 & 93.33 & 93.33 \\
\hline Cosine & 92.50 & 91.67 & 88.33 & 88.33 \\
\hline Correlation & 92.50 & 91.67 & 89.17 & 88.33 \\
\hline Average & 91.94 & 90.56 & 89.86 & 89.44 \\
\hline
\end{tabular}

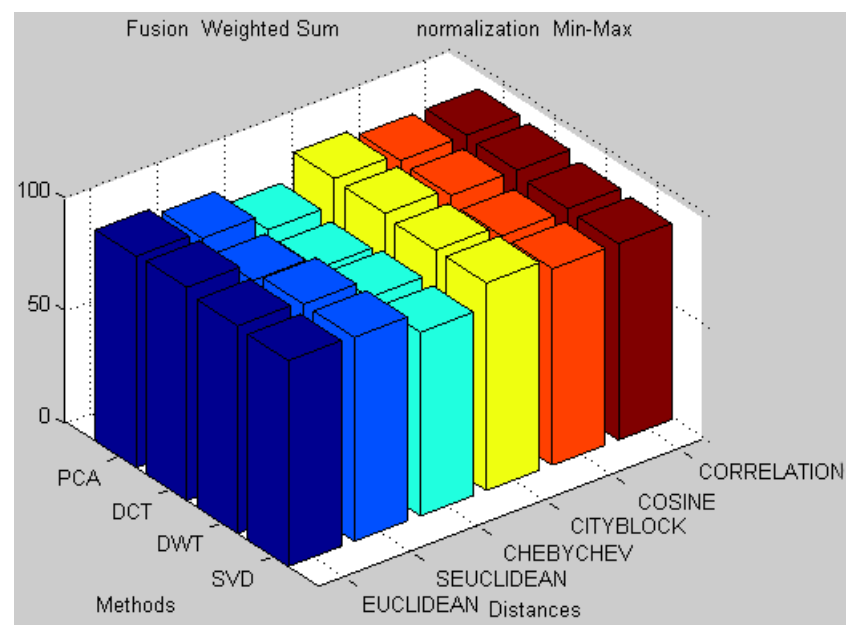

Figure 5. Histograms obtained multimodal recognition rate (face + iris) utilizing normalization (Min-Max) and utilizing (Weighted sum) fusion rules.

Table 5. Obtained recognition rate of multimodal recognition (face + iris) utilizing geometric mean and utilizing fusion rules (Simple sum)

\begin{tabular}{|l|l|l|l|l|}
\hline \multirow{3}{*}{ Rule } & \multicolumn{4}{|c|}{ Normalization (Geometric mean) } \\
\cline { 2 - 5 } & \multicolumn{4}{|c|}{ Simple Sum } \\
\cline { 2 - 5 } & PCA & DCT & DWT & SVD \\
\hline Euclidean & 95.00 & 95.00 & 91.67 & 94.17 \\
\hline Seuclidean & 90.83 & 85.00 & 93.33 & 92.50 \\
\hline Chebychev & 82.50 & 77.50 & 82.50 & 76.67 \\
\hline Cityblok & 98.50 & 95.83 & 94.17 & 91.67 \\
\hline Cosine & 91.67 & 89.17 & 86.67 & 88.33 \\
\hline Correlation & 90.83 & 89.17 & 75.83 & 86.67 \\
\hline Average & 91.38 & 88.61 & 87.36 & 87.16 \\
\hline
\end{tabular}

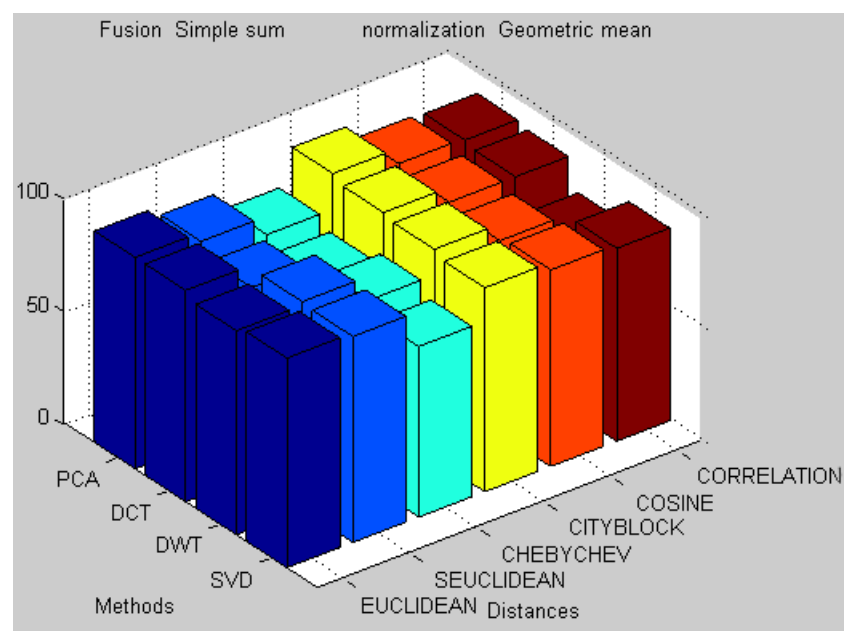

Figure 6. Histograms obtained multimodal recognition rate (face + iris) utilizing geometric mean and utilizing (Simple sum) fusion rules.

Table 6 depicts the assessment results of the multi-modal system using normalization (Geometric mean) and using (Weighted Sum) fusion rules. The PCA and DWT methods gave the best Data recognition rate (95.83\%) for the city block distance. Figure 7 shows the histograms obtained multimodal recognition rate (face + iris) using normalization (Geometric mean) and using (Weighted Sum) fusion rules. We notice that The PCA method gives the best results for extracting the features, and city block distance gives the best results for classification, and new method Geometric mean for normalization. We observe that the performances of the multimodal framework depend on the choice of the methods for extracting the features and the normalization technique as well as the fusion method. For each fusion method there corresponds a specific normalization technique.

Table 6. Obtained recognition rate of multimodal recognition (face + iris) utilizing geometric mean and utilizing fusion rules (Weighted sum)

\begin{tabular}{|l|c|c|c|c|c|}
\hline \multirow{2}{*}{\multicolumn{1}{c|}{ Rule }} & \multicolumn{5}{|c|}{ Normalization (Geometric mean) } \\
\cline { 2 - 6 } & \multicolumn{5}{|c|}{ Weighted Sum } \\
\cline { 2 - 6 } & PCA & DCT & DWT & SVD & Average \\
\hline Euclidean & 95.00 & 94.17 & 93.33 & 92.50 & 93.75 \\
\hline Seuclidean & 88.33 & 84.17 & 90.83 & 91.67 & 88.75 \\
\hline Chebychev & 91.67 & 81.67 & 81.67 & 83.33 & 84.58 \\
\hline Cityblok & 95.83 & 95.00 & 95.83 & 90.83 & 95.59 \\
\hline Cosine & 93.33 & 90.83 & 87.50 & 87.50 & 89.79 \\
\hline Correlation & 93.33 & 91.67 & 80.83 & 86.67 & 88.61 \\
\hline Average & 92.91 & 89.58 & 88.33 & 87.50 & \\
\hline
\end{tabular}




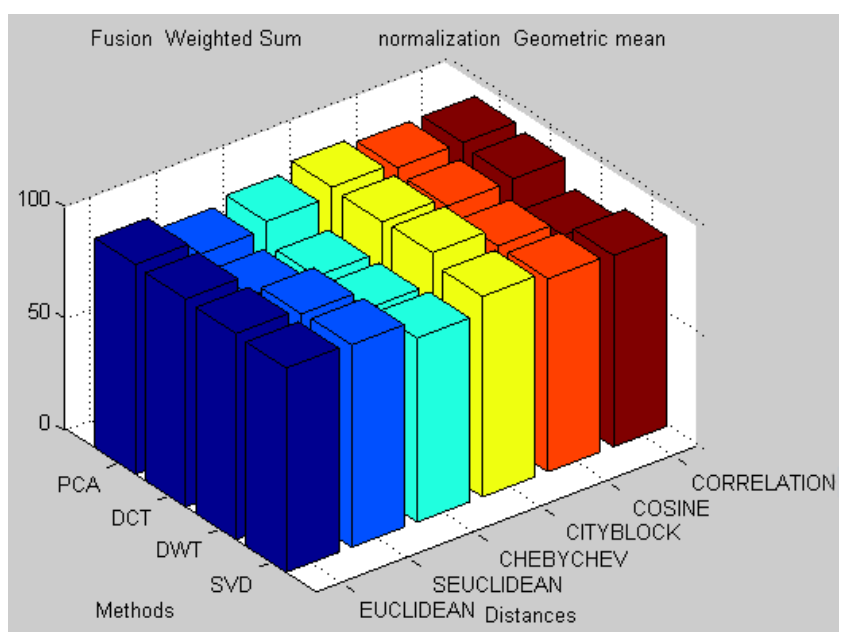

Figure 7. Histograms obtained multimodal recognition rate (face + iris) utilizing geometric mean and utilizing (Weighted sum) fusion rules.

\section{Conclusion}

The biometric systems are becoming more and more widespread in all everyday applications because of the rapid development of new technologies and the constant decline of the cost and the size of the equipment. This study has allowed us to validate the attainability of a framework multimodal biometric by the fusion of two biometric modalities face and the iris. The objective for the researchers was to try to implement modal recognition of more accurate in terms of identification.

In this work, some of the popular methods of face recognition and iris that are PCA, DCT, DWT and SVD are implemented, using the measuring distance (Euclidean, Seuclidian, Chebychev, City block, Cosine and Correlation) and experiments are carried out on the basis of data ORL face and the database of the Iris CASIA (China Institute of Automation). The system multibiometric is introduced as well as since systems uni-biometric fail in some cases. systems multi-modal are used at the level of scores based on two fusion rules simple Sum, Weighted Sum and We have also proposed a new technique for the score normalization to enhance the performance of the system of identification. We found that the multi-modal system using normalization (Geometric mean) and using (simple sum) fusion rules with PCA method and city block distance gave the best Data recognition rate $(98.50 \%)$. We have shown that the performance of a multimodal biometric framework relies upon the choice of the technical normalization; in par- ticular, the proposed normalization technique provides optimal performance when it is coupled with the fusion methods such as the simple Sum.

\section{References}

1. Lim S, Lee K, Byeon O, Kim T. Efficient iris recognition through improvement of feature vector and classifier. ETRI Journal. 2001; 23(2):61-70. https://doi.org/10.4218/ etrij.01.0101.0203

2. Ma L, Wang Y, Tan T. Iris recognition based on multi-channel Gabor filtering. Proceedings Fifth Asian Conference on Computer Vision. 2002; 1:279-83. PMid:11911491

3. Jain AK, Park U. Facial marks: soft biometric for face recognition. 16th IEEE International Conference on Image Processing (ICIP). 2009; p. 37-40. https://doi.org/10.1109/ ICIP.2009.5413921

4. Ibrahim R, Zin ZM. Study of automated face recognition system for office door access control application. IEEE 3rd International Conference on Communication Software and Networks (ICCSN). 2011; p. 132-6.

5. Pillai JK, Patel VM, Chellappa R, Ratha NK. Secure and robust iris recognition using random projections and sparse representations. IEEE Transactions on Pattern Analysis and Machine Intelligence. 2011; 33(9):1877-93. https://doi. org/10.1109/TPAMI.2011.34 PMid:21339529

6. Chen $\mathrm{CH}$, Te Chu C. Fusion of face and iris features for multimodal biometrics. Advances in Biometrics. 2006; 3832:571-80.

7. Liau HF, Isa D. Feature selection for support vector machine-based face-iris multimodal biometric system. Expert Systems with Applications. 2011; 38(9):11105-11. https://doi.org/10.1016/j.eswa.2011.02.155

8. Galbally J, Marcel S, Fierrez J. Image quality assessment for fake biometric detection: application to iris, fingerprint, and face recognition. IEEE Transactions on Image Processing. 2014; 23(2):710-24. https://doi.org/10.1109/ TIP.2013.2292332 PMid:26270913

9. Shahenda S, Shaaban A, Samir E. Multimodal Biometric Systems: A Comparative Study. Arabian Journal for Science and Engineering. 2017; 42(2):443-57. https://doi. org/10.1007/s13369-016-2241-0

10. Raja J, Gunasekaran K, Pitchai R. Prognostic evaluation of multimodal biometric traits recognition based human face, finger print and iris images using ensembled SVM classifier. Cluster Computing. 2018; p. 1-14.

11. Fusion of SVD and DCT-LBP for Face Recognition. Available from: file://C:/Users/a/Downloads/preprints201608.0088.v1.pdf. Date accessed: 2016.

12. Patil MM, Yardi AR. Classification of 3D Magnetic Resonance Images of Brain using Discrete Wavelet 
Transform. International Journal of Computer Applications. 2011; 31(7):0975-8887.

13. Imran M, Rao A, Hemantha Kumar G. Multibiometric Systems: A Comparative Study of Multi-algorithmic and Multimodal Approaches. Procedia Computer Science. 2010; 2:207-12. https://doi.org/10.1016/j. procs.2010.11.026

14. Pairwise distance between pairs of observations. Available from: https://www.mathworks.com/help/stats/pdist.html. Date accessed: 2018.

15. Fusion de la DCT-PCA et la DCT-RLDA pour la Reconnaissance de Visages. Available from: http://www. univ-tebessa.dz/fichiers/univ-blida/taima-fusion-de-laDCT-PCA-et-la-DCT-RLDA-pour-la-reconnaissance.pdf. Date accessed: 2018.

16. Fierrez J, Morales A, Vera-Rodriguez R, Camacho D. Multiple classifiers in biometrics. Part 1: Fundamentals and Review. Information Fusion. 2018; 44:57-64.

17. Statistics and Machine Learning Toolbox. Available from: https://fr.mathworks.com/help/stats/geomean.htm. Date accessed: 2018. 